\title{
Analysis of the effect of target scattering on the operational effectiveness of seeker
}

\author{
Jun-chao Yuan ${ }^{1,}$, Xiao-kuan Zhang ${ }^{1}$, Sheng-yuan WU ${ }^{1}$, Mao Liu ${ }^{1}$ \\ ${ }^{1}$ School of Air Force Engineering University, Xi'an 710051, China \\ a17791252698@163.com
}

\begin{abstract}
The scattering characteristics of targets are important factors influencing the combat effectiveness of the seeker. First, RCS of complex target is calculated by combining PO and MEC based on surface elements. Then, by establishing missile-target intersection model, kill probability and the curve of RCS under different conditions are obtained by simulation. The results show that RCS of the target changes with the change of miss distance and miss azimuth. The changes have a direct impact on the operational effectiveness of the seeker. Severely, seeker will not start.
\end{abstract}

Keywords: electromagnetic scattering, miss distance, miss azimuth

\section{Introduction}

Seeker is an important part of the air defense missile weapon system. Combat effectiveness of seeker is the final index to reflect the terminal effectiveness of guided weapon system. In the period of missile-target intersection, the distance between the seeker and the target is very close. The distance determines that seeker operates near the scattering area of the target. Under different conditions, the target scattering characteristics are different [1] which will affect the operational effectiveness of seeker necessarily. Therefore, the research on the influence of the missile target scattering characteristics on the seeker's combat effectiveness has become a hot topic in Military Science.

The scattering field of the target is obtained by combining PO and MEC based on surface element. Typical missile-target intersection model is established. The paper focuses on influences of the scattering characteristics of the target on the operational effectiveness of the seeker.

\section{Calculation of complex target scattering}

Method of surface elements is an effective method to calculate the scattering of complex targets. The basic idea is to take each surface element in the target mesh model as a scattering point. The total scattering field of the target is considered as the complex vector superposition of the far field scattering field at each observation point. The method of physical optics (PO) is taken to calculate the specular scattering of surface element and the method of equivalent currents (MEC) is taken to calculate the edge diffraction. The total scattering field of target is obtained by superposition of two kinds of scattering fields.

\subsection{Solutions of the incident field [2]}

The amplitude and polarization direction of the incident field of each target scattering unit are different, because the incident wave is an inhomogeneous spherical wave. In order to calculate the scattering, the incident field of each scattering unit should be determined at first. Antenna radiation intensity of far field $E(r, \theta, \varphi)$ is

$$
\boldsymbol{E}(r, \theta, \varphi)=\frac{60 I_{\mathrm{A}}}{r} \boldsymbol{e}_{r} f(\theta, \varphi) e^{-j k r}
$$

In Eq. (1), $I_{\mathrm{A}}$ is aerial current, and $e_{\mathrm{r}}$ is unit position vector.

Normalized antenna pattern function $F(\theta, \varphi)$ is defined as

$$
F(\theta, \phi)=\frac{f(\theta, \phi)}{f_{\max }}=\frac{\left|\boldsymbol{E}_{1}(\theta, \phi)\right|}{\left|\boldsymbol{E}_{\text {max }}\right|}
$$


In Eq. (2), $E_{\max }$ is the intensity of electric field in the direction of maximum radiation. $E_{1}(\theta, \varphi)$ is the intensity of electric field at the same distance in the direction of $(\theta, \varphi)$.

When the electric field intensity $E_{0}$ is in the direction of maximum radiation at the distance $r_{0}$, the amplitude of the radiated electric field at any position $E(r, \theta, \varphi)$ can be obtained by simultaneous Eq. (1) and Eq. (2).

$$
E(r, \theta, \phi)=\boldsymbol{E}_{0} \frac{r_{0}}{r} \boldsymbol{e}_{r} F(\theta, \phi) e^{-j \mathrm{k}\left(r-r_{0}\right)}
$$

$E(r, \theta, \varphi)$ in Eq. (3) is complex number which does not contain the polarization direction of electric field. In this paper, the electric field polarization mode is to take $e_{\mathrm{r}}$ as the propagation direction of electromagnetic wave in the spherical coordinate system. $e_{\theta}$ and $e_{\phi}$ are considered as two bases of orthogonal polarization. Then, arbitrary elliptical polarization $p_{\mathrm{e}}$ can be expressed as

$$
\boldsymbol{p}_{\mathrm{e}}=\boldsymbol{e}_{\theta} \cos \theta_{\mathrm{R}}+\boldsymbol{e}_{\phi} \sin \theta_{\mathrm{R}} e^{\left(j \delta_{\mathrm{R}}\right)}
$$

In Eq. (4), $\theta_{\mathrm{R}}$ is the intersection angle between $p_{\mathrm{e}}$ and $e_{\theta} \cdot \delta_{\mathrm{R}}$ is the phase angle which shows that $e_{\phi}$ component of $p_{\mathrm{e}}$ advances $e_{\theta}$ component. The direction of the incident electric field can be obtained when coordinates of each scattering unit are known. The incident electric field $E_{\mathrm{i}}$ of $\mathrm{i}^{\text {th }}$ scattering unit with coordinates $\left(r_{i}, \theta_{i}, \varphi_{i}\right)$ can be expressed as

$$
\boldsymbol{E}_{i}=E(r, \theta, \varphi) \boldsymbol{p}_{e}
$$

\subsection{Physical optics method}

Physical optics method replaces the scatterer with the equivalent current on the scatterer. The scattering field is obtained by the approximation and integration of surface. The basic starting point is Stratton - chu integral equation.

$$
\begin{aligned}
\boldsymbol{E}_{s}= & \frac{j k}{4 \pi} \frac{e^{-j k r}}{r} \int_{s} \boldsymbol{s} \times\left(\boldsymbol{M}_{\mathrm{s}}+\eta_{0} \boldsymbol{s} \times \boldsymbol{J}_{\mathrm{s}}\right) . \\
& \exp \left[j k \boldsymbol{r}^{\prime} \cdot(\boldsymbol{s}-\boldsymbol{i})\right] d s
\end{aligned}
$$

In Eq. (6), $\overrightarrow{\mathbf{i}}$ and $\overrightarrow{\mathbf{s}}$ are unit vectors of incident and scattering direction. $r$ is the position of field point. $\overrightarrow{\mathbf{r}}^{\prime}$ stands for the position vector of source point. $s$ indicates the illuminated part of object. $\overrightarrow{\mathbf{J}}_{\mathrm{s}}$ and $\overrightarrow{\mathbf{M}}_{\mathrm{s}}$ stand for equivalent surface current and equivalent edge currents on $S$.

For the conductor target, equation can be simplified as

$$
\boldsymbol{J}_{\mathrm{s}}=2 \boldsymbol{n} \times \boldsymbol{H}^{i} ; \boldsymbol{M}_{\mathrm{s}}=0
$$

$n$ stands for unit vector in the normal direction of the surface. $H_{\mathrm{i}}$ stands for the incident magnetic field on the surface.

\subsection{Equivalent electromagnetic flow method [3]}

Edge diffraction field of any wedge, the edge of which is C, can be expressed as follows.

$$
\begin{gathered}
\boldsymbol{E}_{d}=\frac{j k e^{-j k r}}{4 \pi r} \int_{C}\left\{\eta_{0} \boldsymbol{s} \times\left[\boldsymbol{s} \times \boldsymbol{J}\left(\boldsymbol{r}^{\prime}\right)\right]+\right. \\
\left.\boldsymbol{s} \times \boldsymbol{M}\left(\boldsymbol{r}^{\prime}\right)\right\} e^{j k s \cdot r^{\prime}} d l
\end{gathered}
$$

In the above equation, $\mathrm{J}\left(r^{\prime}\right)=I_{\mathrm{e}}\left(r^{\prime}\right) t$ and $\mathrm{M}\left(r^{\prime}\right)=I_{\mathrm{m}}\left(r^{\prime}\right) t$ are equivalent edge current and equivalent marginal magnetic current separately. $t$ is the tangential unit vector of C. $r$ is the location of field point. $r^{\prime}$ is position vector of edge element.

\subsection{GRCS}

Generalized RCS considering receiving polarization is defined as follows [4].

$$
G R C S=4 \pi\left|\boldsymbol{r}_{0}-\boldsymbol{r}\right|^{2} \frac{\left|\boldsymbol{E}_{s}(\boldsymbol{r}) \cdot \boldsymbol{e}_{r}\right|^{2}}{\left|\boldsymbol{E}_{i}\left(\boldsymbol{r}_{0}\right)\right|^{2}}
$$


In Eq. (9), $r_{0}$ is geometric center coordinates of scatter. $r$ is the phase center of receiving antenna. $e_{\mathrm{r}}$ is the polarization of receiving antenna.

\section{Operational effectiveness of Seeker}

The operation process of the seeker is that in the projectile encounter section, the warhead kill material can hit the target most likely through the timely initiation of the warhead. In other words, dynamic killing zone of warhead and antenna pattern of seeker try to overlap in order to maximize the hit of target and get the satisfied destruction effect. The operational effectiveness of the seeker is an indicator of the coordination between them. Fig. 1 gives a schematic diagram of the ideal operation of the seeker.

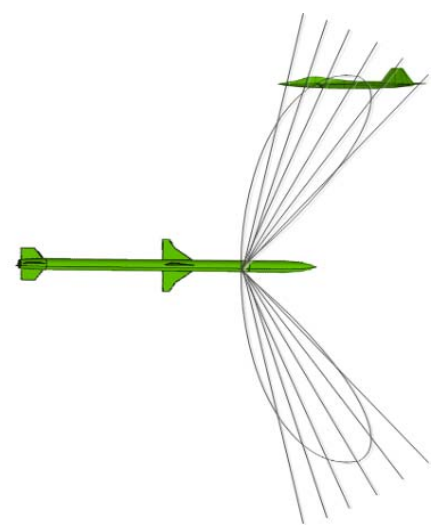

Fig. 1 Ideal operation of the seeker

\subsection{Conditional kill probability}

Conditional kill probability $P_{\mathrm{df}}(\rho, \theta)$ is defined under given conditions of miss distance $\rho$ and miss azimuth $\theta$.

$$
P_{\mathrm{df}}(\rho, \theta)=P_{\mathrm{f}}(\rho, \theta) \int_{x_{\min }}^{x_{\max }} P_{\mathrm{d}}\left(\left.x\right|_{\rho, \theta}\right) f_{\mathrm{f}}\left(\left.x\right|_{\rho, \theta}\right) d x
$$

$P_{\mathrm{f}}(\rho, \theta)$ stands for the starting probability of seeker. $P_{\mathrm{d}}\left(\left.x\right|_{\rho, \theta}\right)$ is the kill probability of target when the seeker is under the condition of $(x, \rho, \theta)$ and warhead explores. $f_{\mathrm{f}}\left(\left.x\right|_{\rho, \theta}\right)$ is the density function of the relative trajectory of start point $x$. The paper assumes that the warhead of air defense missile uses fragmentation warhead, and simple rod kill model is chosen as the target vulnerability model. Specific definitions and calculation methods of the parameters can be found in [5]. Parameters in simulation are given. Although the conditional kill probability is a statistical index, it is determined for the start point $x$ during one determined intersection process. Thus, the paper takes $P_{\mathrm{df}}(\rho, \theta)$ as a quantitative index to describe the operational effectiveness of seeker.

\subsection{Start point of seeker}

The start point of the seeker is the position of the warhead when the target is sensed. In the process of missile-target rendezvous, the radiation of the antenna beam to the target can be regarded as the scanning process of the narrow beam to the target. Therefore, the illumination area of the target is changed, when the scattering characteristics of the target are changed, and the echo intensity is also changed [6]. It is assumed that when the echo intensity exceeds sensitivity of the seeker, the seeker starts absolutely. Then, the central position of the warhead is the starting point of the seeker. Sensitivity of the seeker is defined as

$$
S_{f 0}=10 \lg \left(P_{t} / P_{r \min }\right)
$$

In the above equation, $P_{t}$ is transmitting power of the seeker. $P_{\mathrm{rmin}}$ is minimum starting power of the seeker.

$$
\frac{P_{t}}{P_{r}}=\frac{(4 \pi)^{3} r^{4}}{G_{t} G_{r} \sigma \lambda^{2}}
$$


According to the distance formula of seeker, $\sigma$ is equivalent scattering cross section of the target. $\lambda$ is length of operating wave of the seeker. $r$ is the distance between missile and target. $G_{t} 、 G_{\mathrm{r}}$ are transmitting and receiving antenna gain respectively.

The target GRCS achieved in section 2.4 is put into equation (12) and compared with the relative sensitivity of seeker $S_{\mathrm{f}_{0}}$. The position of start point of the seeker can be obtained under given conditions.

\subsection{Missile-target intersection model}

The calculation of target scattering characteristics of the seeker is the problem of dynamic simulation about missile-target intersection. The target and the missile can be approximated as a uniform linear flight due to the short duration of operation of the seeker. Thus, the relative velocity coordinate system is choose to study the problem. In the relative velocity coordinate system, the direction of relative velocity $V_{c l}$ is the positive direction of $\mathrm{x}$ axis. The $\mathrm{Y}$ axis is taken in the vertical plane and upward direction is positive. $\mathrm{Z}$ axis is set in the horizontal plane and has right spiral relationship with $\mathrm{x}$ axis and y axis. Miss distance $\rho$ is defined as distance between the hit point and the target in the missing plane. The missing plane is defined as the plane which is over the target and is perpendicular to the direction of missile-target relative velocity. The relationship is shown in fig. 2.

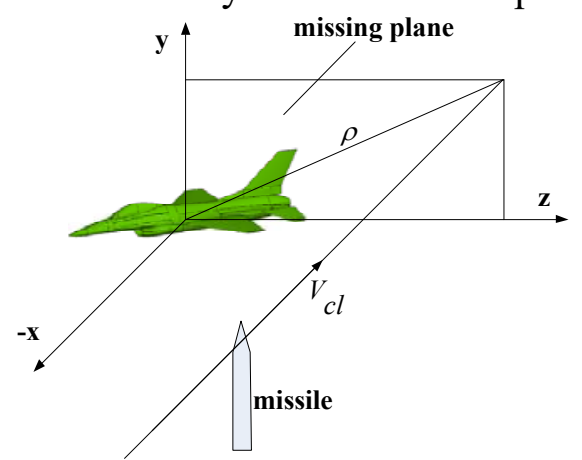

Fig. 2 Relative velocity coordinate system

\section{The results and analysis of simulation}

Fig. 3 describes the process of simulation of the seeker's operation. First, the relative parameters such as the relative position and relative velocity of missile-target are obtained by the model of missile-target intersection. Then, the GRCS of the target is obtained by vector synthesis, using the scattering field of each scattering unit achieved from PO+MEC calculation model of scattering. Scattering information of the target is used to judge the start. If the start condition is reached, the kill probability of the warhead under the position of the starting point is calculated. Otherwise, simulation at next time will be calculated.

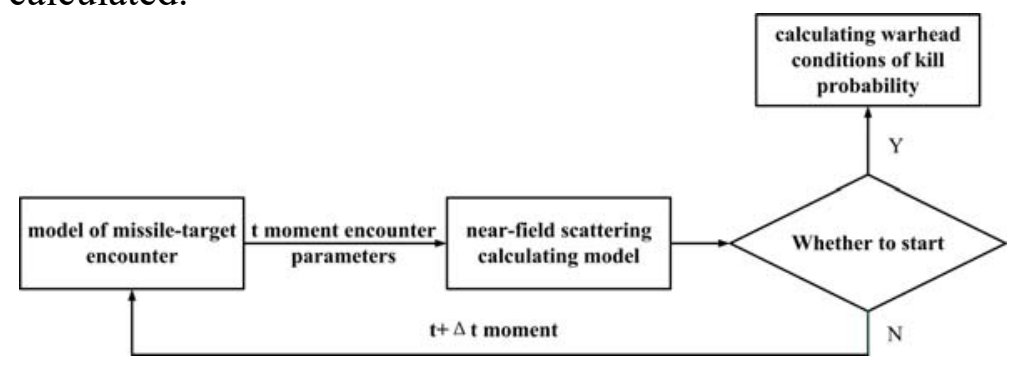

Fig. 3 Simulation process of seeker's operation

In the simulation, relative velocity is $V_{c l}=1000 \mathrm{~m} / \mathrm{s}$. The sensitivity of the seeker is taken as $S_{\mathrm{f}_{0}}=75 \mathrm{~dB}$ [7]. Fig. 4 shows the conditional kill probability of warhead when missile approaches target from different rendezvous trajectory. 


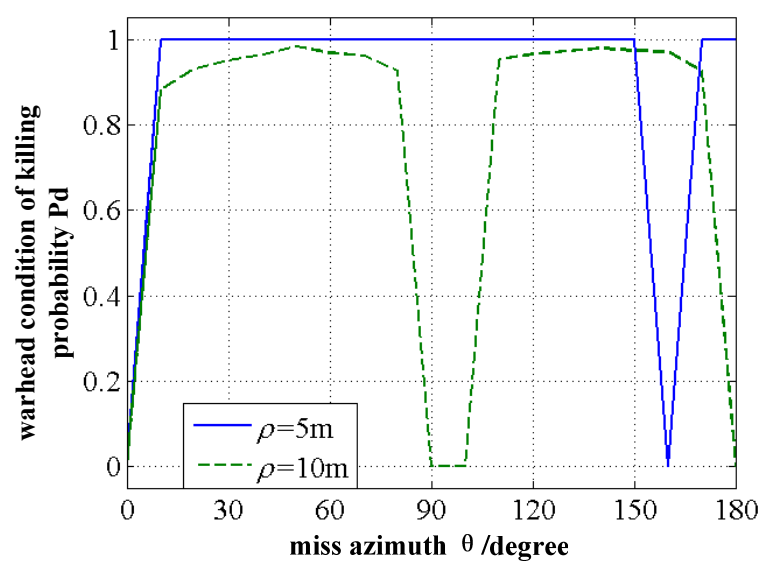

Fig. 4 The change of seeker's operational effectiveness with the change of miss azimuth

According to fig. 4, the reduction of the miss distance can effectively improve the conditional kill probability of warhead and the operational effectiveness of the seeker. The conditional kill probability of warhead $P_{\mathrm{d}}$ is close to 1 when the miss distance of trajectory is between $5 \mathrm{~m}$ and $10 \mathrm{~m}$. It means that the seeker has ideal operational effectiveness. The $P_{\mathrm{d}}$, when $\rho=10 \mathrm{~m}$, is a little smaller than that when $\rho=5 \mathrm{~m}$. Then reason is that when the distance between missile and target is far, the velocity of fragments is reduced by air resistance. $P_{\mathrm{d}}$ equals to 0 under some conditions, for example that the miss distance is $10 \mathrm{~m}$ and the miss azimuth is $0^{\circ}, 90^{\circ}, 100^{\circ}$ and $180^{\circ}$. Another example is that the miss distance is $5 \mathrm{~m}$ and the miss azimuth is $160^{\circ}$. It is because that the seeker does not start. Three trajectories are selected to illustrate in table 1. The intersection parameters of different trajectories are also given in table 1. Fig. 5 shows the RCS curves of three intersection trajectories. The remaining time is flight time when the missile reaches the missing plane. When $t=0$, missile position corresponds to the miss point.

Table 1 Parameters of different trajectories

\begin{tabular}{|c|c|c|c|c|c|}
\hline Prajectory & $\rho, \theta$ & $\begin{array}{c}\text { Initial time } \\
t_{1} / \mathrm{ms}\end{array}$ & $\begin{array}{c}\text { Starting time } \\
t_{2} / \mathrm{ms}\end{array}$ & $\begin{array}{c}\text { Starting point } \\
\mathrm{x} / \mathrm{m}\end{array}$ & $\begin{array}{c}\text { Conditional kill } \\
\text { probability of } \\
\text { warhead }\end{array}$ \\
\hline Trajectory 1 & $5 \mathrm{~m}, 90^{\circ}$ & 2.648 & 2.648 & -2.648 & 1.000 \\
\hline Trajectory 2 & $10 \mathrm{~m}, 80^{\circ}$ & 4.814 & 2.388 & -2.388 & 0.927 \\
\hline Trajectory 3 & $10 \mathrm{~m}, 90^{\circ}$ & 4.789 & Not start & None & 0 \\
\hline
\end{tabular}




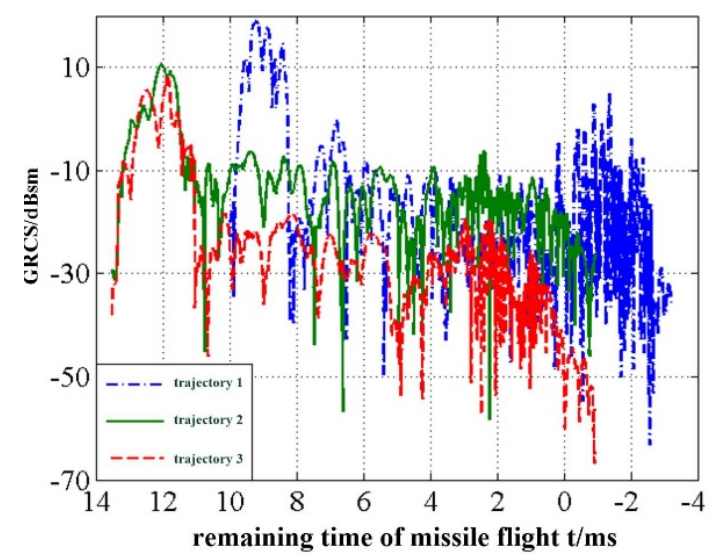

Fig. 5 Comparison of RCS in different trajectories

In trajectory 1 , while $t_{1}=t_{2}$ two time are different in trajectory 2 . The reason is that the precondition of seeker's initiation is that the echo's power does not reach the seeker's sensitivity. According to fig. 5 , when the GRCS of target is small during the period that the sensitivity of the seeker reaches the condition of start, the start of the seeker will be delayed(such as trajectory 2) or the seeker will not start(such as trajectory 3). Combining fig. 4, although the maximum value of GRCS of the target is much larger compared with other trajectories with the same miss distance under the condition that the miss distance is $10 \mathrm{~m}$ and the miss azimuth is $90^{\circ}$, the seeker cannot start. It can be seen that the maximum GRCS value is not the key factor that determines the start of the seeker. The influence of the GRCS of the whole missile rendezvous process on the launch of the seeker should be fully considered.

Based on the analysis, in order to ensure that the seeker can start effectively to achieve high combat effectiveness, the target RCS should be taken as the design index and the scattering characteristics of the whole rendezvous process should be taken into account in the design of seeker's sensitivity.

\section{Conclusion}

The paper analyzes impacts of scattering characteristics on the operational effectiveness of the seeker through simulation. According to the results, RCS of target has an important impact on the operational effectiveness of the seeker. Therefore, in order to ensure the reliable start of the rendezvous trajectory, it is necessary to consider the influence of the RCS and the optimization scheme of the combination of the missile and target rendezvous in the design of the seeker launching scheme.

\section{References}

[1] Wu Ling and Xia Yingqing, The Impact of Antenna Pattern on Targets Near-field Scattering Characteristics, Journal of HuaZhong Normal University, Natural Sciences, Vol. 48 (Nat.Sci, 2014) pp. 339-342

[2]Wang Xinwen, Li Yanping and Li Ping, Microwave Technology and Antenna, Electronic Industry Press (2011)

[3]Zhao Weijiang, Gong Shuxi and Liu Qizhong, New Expressions for Fringe Current Components of Equivalent Edge Currents, Acta Electronica Sinica, Vol. 30 (2002)pp.1340-1343

[4]Gao Hongwei, Gong Li and Sheng xinqing, Computation of Near-field Scattering by 3-D Complex Target, Transactions of Beijing Institute of Technology, Vol. 34 (2014)pp. 88-91.

[5]Liu Tong, The Lethality Assessment Method of Air-Denfense Missile Warhead[D], Nanjing University of Science and Technology, 2004.

[6]Nie Jiankun, Study on the Related Problems of the Near EM field of Missile-borne Antenna, Master's thesis, Xidian University (2012)

[7]Zhao Qi, Chen Ning, Fei Yuanchun. Modeling and Realizing of Seeker Target Echo's Simulation[J], Joural of system simulation, 2008, 20(10): 2674-2681. 